\title{
Preface-Special Issue on Recent Developments in Operations Research: Theory and Applications
}

\author{
Wuyi Yue ${ }^{1} \cdot$ Hsing Luh ${ }^{2} \cdot$ Duan $\mathrm{Li}^{3}$ \\ Published online: 30 October 2020 \\ ( ) Operations Research Society of China, Periodicals Agency of Shanghai University, Science Press, and \\ Springer-Verlag GmbH Germany, part of Springer Nature 2020
}

This issue of Journal of the Operations Research Society of China is a special one focusing on recent developments in Operations Research (OR): Theory and Applications. This special issue makes a significant contribution to OR in a rapidly changing environment.

All participants of the 13th International Symposium on Operations Research and its Applications were cordially invited to submit their papers to this special issue. It was also open to all researchers of OR and its related areas. Eight papers were accepted for publication after rigorous reviewing processes. These papers included recent theoretical and applied contributions in various fields of $\mathrm{OR}$ and related topics. Short descriptions of these submissions are as follows.

Tatsuo Oyama presents an overview paper: Looking for and Aiming for an Asian OR Applicable to the Public Sector. In this paper, at first, the history of OR, especially its application to public sectors is briefly described; then the characterization of using OR as a scientific method for decision making is presented. Further, OR activities in Japan are introduced, emphasizing three major roles: (i) quantitative data analysis, (ii) mathematical modeling analysis, and (iii) theory building analysis. An example for each of these three roles is then described.

Xiushuang Wang, Jing Zhu, Shunfu Jin, Wuyi Yue, and Yutaka Takahashi present a virtual machine allocation scheme with a sleep-delay, establish and demonstrate a corresponding mathematical model for the performance analysis and evaluation of network resource in a cloud environment. Further, a two-dimensional Markov chain

\footnotetext{
$\bowtie \quad$ Wuyi Yue

yue@konan-u.ac.jp

Hsing Luh

slu@nccu.edu.tw

Duan Li

dli226@cityu.edu.hk

1 Department of Intelligence and Informatics, Konan University, Kobe, Japan

2 National Chengchi University, Taipei, China

3 City University of Hong Kong, Hong Kong, China
} 
is constructed to derive numerically the average latency of tasks and the energysaving degree of the system in the steady state. Moreover, the authors investigate the Nash equilibrium behavior and the socially optimal behavior of tasks and carry out an improved adaptive genetic algorithm to obtain the socially optimal arrival rate of tasks. A pricing policy for tasks to maximize the social profit when managing the network resource is also presented.

Ning Zhang, Chang-Jun Yu, and Fu-Sheng Xie consider a class of optimal control problems in which the dynamic systems are time-delay switched systems with the delay as a function of time. By applying the control parameterization method, the control heights and switching times become decision variables that need to be optimized. This paper introduces a time-scaling transformation technique to transform the original system into an equivalent system, which is defined on a new time horizon with fixed switching times. Further, the gradients of the objective and constraint functions with respect to the control heights and durations are derived based on the relationship between the original time scale and the newtime scale. Finally, the new problem is solved by using a gradient-based optimization approach.

Hozumi Morohosi presents a network-based optimization model for planning the search path by using multiple unmanned aerial vehicles (UAVs) to search a victim in a disaster relief operation. The author proposes a way of using multiple UAVs for searching a disaster-stricken area and estimates the number of UAVs necessary for the search. A heuristic algorithm is devised to solve the optimization model and applied to the problem instances selected from potential hazard areas in Japan. The computational result shows that a relatively fewer number of UAVs would be required to cover the designated area in most cases. Moreover, this paper analyzes the relationship between the search areas and the number of UAVs necessary for searching via regression methods.

Bhuwan Chandra Joshi, Shashi Kant Mishra, and Pankaj Kumar investigate semi-infinite mathematical programming problems with equilibrium constraints (SIMPPEC) using generalized convexity in their paper. By using the notion of convexificators, the authors establish sufficient optimality conditions for the SIMPPEC and formulate Wolfe and Mond-Weir-type dual models for the SIMPPEC under the invexity and generalized invexity assumptions. Moreover, weak and strong duality theorems are established that relate the SIMPPEC and two dual programs in the framework of convexificators.

Kun-Lun Wang, Chen Fei, and Wei-Yin Fei construct an optimal principal (male)-agent (female) contract model based on the principal's expected profit and the agent's expected utility function by using the sublinear expectation theory under Knightian uncertainty. Further, in this paper, the Hamilton-Jacobi-Bellman equation is deduced by using the optimality principle under sublinear expectation while the smoothness viscosity condition of the principal-agent optimal contract is given. Moreover, the continuation value of the agent is taken as the state variable to characterize the optimal expected profit of the principal, the agent's effort and the consumption level under different degrees of Knightian uncertainty. Finally, behavioral economics is used to analyze the simulation results.

Yuji Matsuo and Tatsuo Oyama propose a hybrid forecasting model for the shortterm electric load forecasting using artificial neural network (ANN), discrete Fourier 
transformation (DFT) and principal component analysis (PCA) techniques in order to attain higher prediction accuracy. Firstly, this paper estimates the Fourier coefficients by using DFT to predict the next-day load curve with an ANN and obtain approximate load curves by applying the inverse discrete Fourier transformation. Then, approximate curves, combined with other input variables, are applied to the ANN to predict the next-day hourly load curves. Furthermore, PCA scores to obtain approximate load curves in the first step, which are again applied to the ANN in the second step, are predicted.

Megumi Yamamoto, Nobuo Umemura, and Hiroyuki Kawano develop a Moodle plug-in, which is an automated essay scoring support system for the evaluation of university students' essays. This system evaluates essays based on a rubric which has five evaluation viewpoints, namely "Content, Structure, Evidence, Style, and Skill". This paper proposes to construct the comprehensive Japanese vocabulary difficulty level dictionaries using Japanese Wikipedia as the corpus. Further, the paper applies Latent Dirichlet Allocation (LDA) to the Wikipedia corpus and determines the word appearance probability as an index of word difficulty. Moreover, the paper uses the TF-IDF (Term Frequency-Inverse Document Frequency) value instead of the LDA value of the words to construct highly comprehensive Japanese vocabulary difficulty level dictionaries.

September, 2020

Acknowledgements As guest editors, we would like to thank all the authors who have shown interest and have contributed to this special issue. We thank all the reviewers who accepted the review invitation to provide their expert and constructive comments to improve the quality of the papers. We thank the Editorsin-Chief of Journal of the Operations Research Society of China, Ya-Xiang Yuan and the staff of the editorial office for their interest and continuous support. We hope that the readers will find the contributions of this special issue enjoyable and inspiring. 